\title{
Real-Time Tree-Foliage Surface Estimation Using a Ground Laser Scanner
}

\author{
Jordi Palacín, Member, IEEE, Tomàs Pallejà, Marcel Tresanchez, Ricardo Sanz, Jordi Llorens, \\ Manel Ribes-Dasi, Joan Masip, Jaume Arnó, Alexandre Escolà, and Joan Ramon Rosell
}

\begin{abstract}
The optimization of most pesticide and fertilizer applications is based on overall grove conditions. In this paper, we propose a measurement system to estimate the foliage surface of a tree crop. The system is based on a ground laser scanner that estimates the volume of the trees and then extrapolates their leaf area using simple and fast algorithms to allow true real-time operation. Tests with pear trees demonstrated that the relation between the volume and the foliage can be interpreted as linear with a coefficient of correlation $(R)$ of 0.81 , and the foliage surface can be estimated from this volume with an average error less than $6 \%$.
\end{abstract}

Index Terms-Agrochemical dose control, foliage estimation, ground laser scanner, light detection and ranging (LIDAR), remote sensing, volume estimation.

\section{INTRODUCTION}

$\mathbf{T}$ RADITIONAL pesticide and fertilizer application in the treatment of groves is based on uniform application, which causes significant material loss (Fig. 1) [1]. The measurement of the overall grove conditions can be used to optimize the application, to provide economic benefits for growers, and to reduce pollution in the environment [2]. The measurement of treecanopy structural characteristics, such as volume and foliage, can be carried out by means of several detection principles, such as image-analysis techniques, stereoscopy photography, analysis of the light spectrum, the infrared thermograph, ultrasonic sensors, and light detection and ranging (LIDAR) laser sensors.

Image-based canopy measurement requires elaborate algorithms and huge computational resources to allow real-time operation [3]. Ultrasonic sensors have been used to measure canopy volume for pesticide applications [4]-[7], although the divergence angle of the ultrasonic waves limits the spatial resolution and the accuracy of the measurements. LIDAR is a remote-sensing technique based on the measurement of the time a laser pulse takes between the sensor and a target and has the advantage that the beam can be very thin and diverges very little [8]; however, it only reports information about the external surface of the trees [9]. In recent years, LIDAR has

Manuscript received June 30, 2006; revised March 20, 2007. This work was supported in part by the Spanish Comisión Interministerial de Ciencia y Tecnología, under Agreement AGL2002-04260-C04-02.

J. Palacín, T. Pallejà, and M. Tresanchez are with the Department of Informatics and Industrial Engineering, University of Lleida, 25001 Lleida, Spain (e-mail: palacin@iee.org).

R. Sanz, J. Llorens, M. Ribes-Dasi, J. Masip, J. Arnó, A. Escolà, and J. R. Rosell are with the Department of Agro-Forestry Engineering, University of Lleida, 25198 Lleida, Spain.

Color versions of one or more of the figures in this paper are available online at http://ieeexplore.ieee.org.

Digital Object Identifier 10.1109/TIM.2007.900126

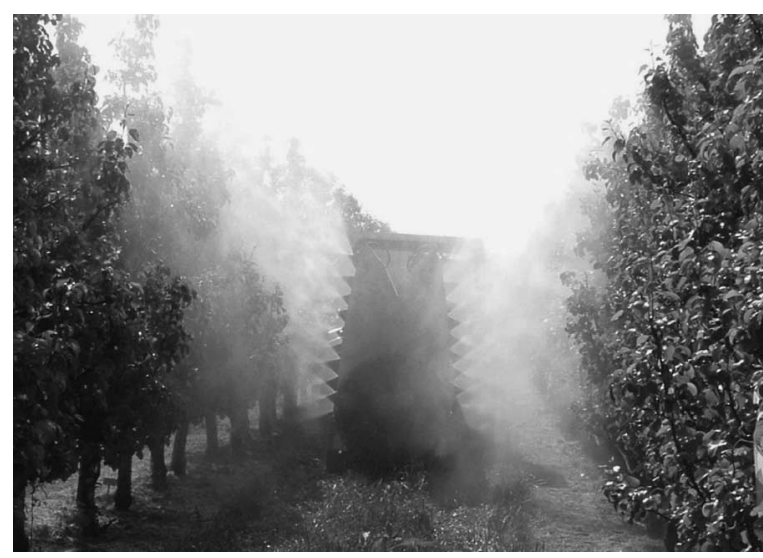

Fig. 1. Pesticide application.

been widely used for the measurement of environmental target parameters in forests and agricultural characterization. The canopy height [10] and tree height and volume [11] were estimated using an airborne LIDAR altimeter. Walklate et al. [12] used the LIDAR to study the relationship between orchardtree-crop structure and performance characteristics of an axial fan sprayer with good correlation between spray deposit and some measure of the foliage density. Tumbo et al. [6] compared the performance of ultrasonic and laser sensors for the measurement of citrus-canopy volume, obtaining good correlations with manual measurements. Recently, Wei and Salyani [13], [14] used a terrestrial LIDAR to measure tree height, width, and volume in developing a set of experiments to evaluate the repeatability and accuracy of the measurements and to obtain a coefficient variation of $5.4 \%$ and a relative error of $4.4 \%$ in the estimation of the volume but without real-time capabilities. These experiments were partially reproduced in the study in [15] with similar results.

The pesticide and fertilizer application can be optimized in two ways: adjusting the overall dose to guarantee uniform application or having an estimate of the foliage surface of the surrounding trees to make adequate the application dose. Normally, uniform application requires an estimate of the real displacement of the sprayer to adjust the overall dose. However, in practice, the uniform dose is accomplished by fixing a dose and a uniform speed in the tractor unit for the entire grove because the tractor has enough power to guarantee a certain degree of uniformity in the speed in all applications, even when using a very simple speed-control loop. This paper is focused on the second alternative: the development of a measurement system for real-time foliage estimation that could be incorporated into 


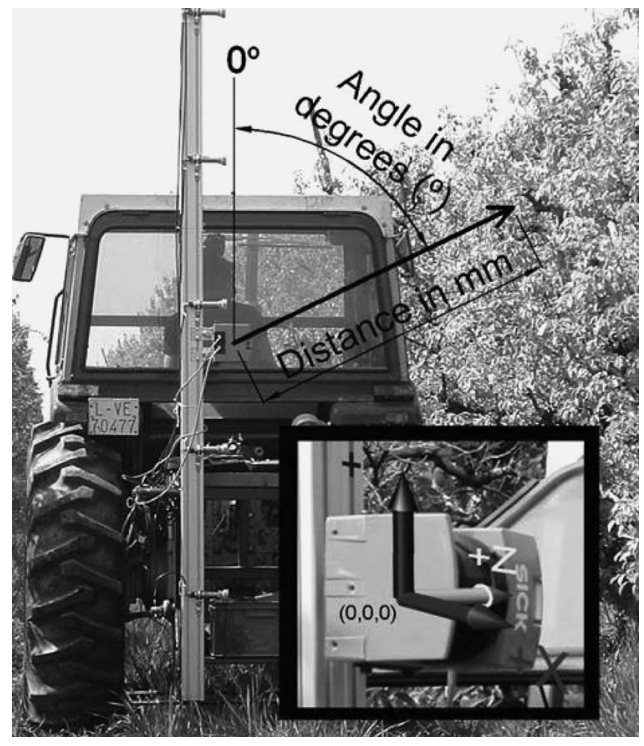

Fig. 2. LIDAR attached to a tractor unit.

any kind of fertilizer or pesticide machine with dose-control capabilities to adjust the quantity of applied product according to crop characteristics.

The main hypothesis of this paper is that there is a relation between the volume and the foliage surface of the trees. With this relation, a measurement of the volume of the trees could be used to estimate their foliage surface and then optimize pesticide and fertilizer application. The fertilizer is usually applied directly to the ground, and only an overall estimation of the entire surrounding foliage surface is enough to control the application. The pesticide is usually applied directly to the trees (Fig. 1) using the same dose for the entire tree and grove, although the application machines normally have the capability to change the dose, depending on the height. Therefore, it could be very useful to have an estimation of the foliage surface at different heights. In this paper, a pear tree grove is the case example, and the estimation of the foliage surface and the real-time capabilities are the main improvement related to the previous work.

This paper is structured as follows. In Section II, we describe our approach; in Section III, the experimental validation of the measurement system is presented; in Section IV, the obtained results are discussed, and some improvements are proposed. Finally, this paper ends with the main conclusions.

\section{Materials AND Methods}

\section{A. Measurement System}

The measurement system is based on a 2-D LIDAR. Fig. 2 shows the LIDAR attached to a tractor unit, and Fig. 3 shows the schema of the complete system that is composed by the LIDAR and 1) a computer for data storage and offline data processing or 2) a dedicated programmable automation controller for realtime tree-foliage estimation. The LIDAR used is a low-cost general-purpose LMS-200 model (Sick, Düsseldorf, Germany) [16]; a fully automatic divergent laser scanner based on the measurement of time-of-flight [17], [18] with an accuracy of $\pm 15 \mathrm{~mm}$ in a single-shot measurement and 5-mm standard deviation in a range of up to $8 \mathrm{~m}$. The time between the transmission and the reception of the pulsed near-infrared laser beam is used to measure the distance between the scanner and the reflecting object surface. The laser beam is deflected by a rotating mirror turning at $4500 \mathrm{r} / \mathrm{min}(75 \mathrm{r} / \mathrm{s})$, which results in a fan-shaped scan pattern where the maximum scanning angle is $180^{\circ}$. The angular resolution is selectable at $1^{\circ}, 0.5^{\circ}$, or $0.25^{\circ}$, making 181,361 , and 400 measures, respectively, at full scanning range with a response time of 13,26 , and $53 \mathrm{~ms}$, respectively. The LMS-200 has a standard RS232 serial port for data transfer with a rate selectable at 9.6, 19.2, or 38.4 KBd and a nonstandard RS422 serial port with a transfer rate of $500 \mathrm{KBd}$ using a specific RS422 card.

The LIDAR is used to obtain vertical slices of the surface of the tree. The raw data generated by the LMS-200 can be configured in three different modes: polar, rectangular, and reflexivity. In polar mode, the raw data are composed only of a sequence of radial distances; in rectangular mode, the data are composed of a pair of relative distances $[X, Y]$; and in reflexivity mode, the data are composed of a sequence of the index of measured reflexivity. In all modes, the first value of the sequence corresponds to the initial measurement angle (normally $0^{\circ}$ ), and the angle of the following values has an increment which depends on the angular resolution selected. The LMS-200 always scans at full speed, storing the information in an internal buffer. The LMS-200 can operate in a singlescan mode, sending the data corresponding to one slice when requested, or in continuous-scan mode, taking and sending slices until the control unit stops this mode. Additionally, the LMS-200 can be configured to internally average a number of consecutive scans. This mode is very useful for noise reduction in a static environment but has no utility in this case with a dynamic environment.

For the proposed application, the LIDAR was configured for nonaveraged single-scan polar-distance measurement with a range up to $8 \mathrm{~m}$ and with an angular resolution of $1^{\circ}$ in a range of $\left[0^{\circ}, \ldots, 180^{\circ}\right]$. The external communication was performed through the RS232 serial port at $38.4 \mathrm{~kb} / \mathrm{s}$. In the development stage, the LIDAR was interfaced to a computer for data recording and offline processing using a graphic interface developed in MatLab (The Mathworks Inc, Natick, MA). In the final test stage, the LIDAR was interfaced to a Compact FieldPoint programmable automation controller (National Instruments Corporation, Austin, TX) for real-time operation.

\section{B. Raw Data Preprocessing}

Figs. 4 and 5 show the top and front views of the scanning scenario that is composed by a tree row line and a LIDAR following a trajectory parallel to a tree line, where

$\theta \quad$ angular resolution of the scans;

$j \quad$ beam index into the scan, starting in the vertical position;

$i \quad$ scan index;

FTS forward travel speed of the LIDAR;

$D_{\mathrm{i}} \quad$ distance between the LIDAR travel line and the tree row line for the scan $i$; 


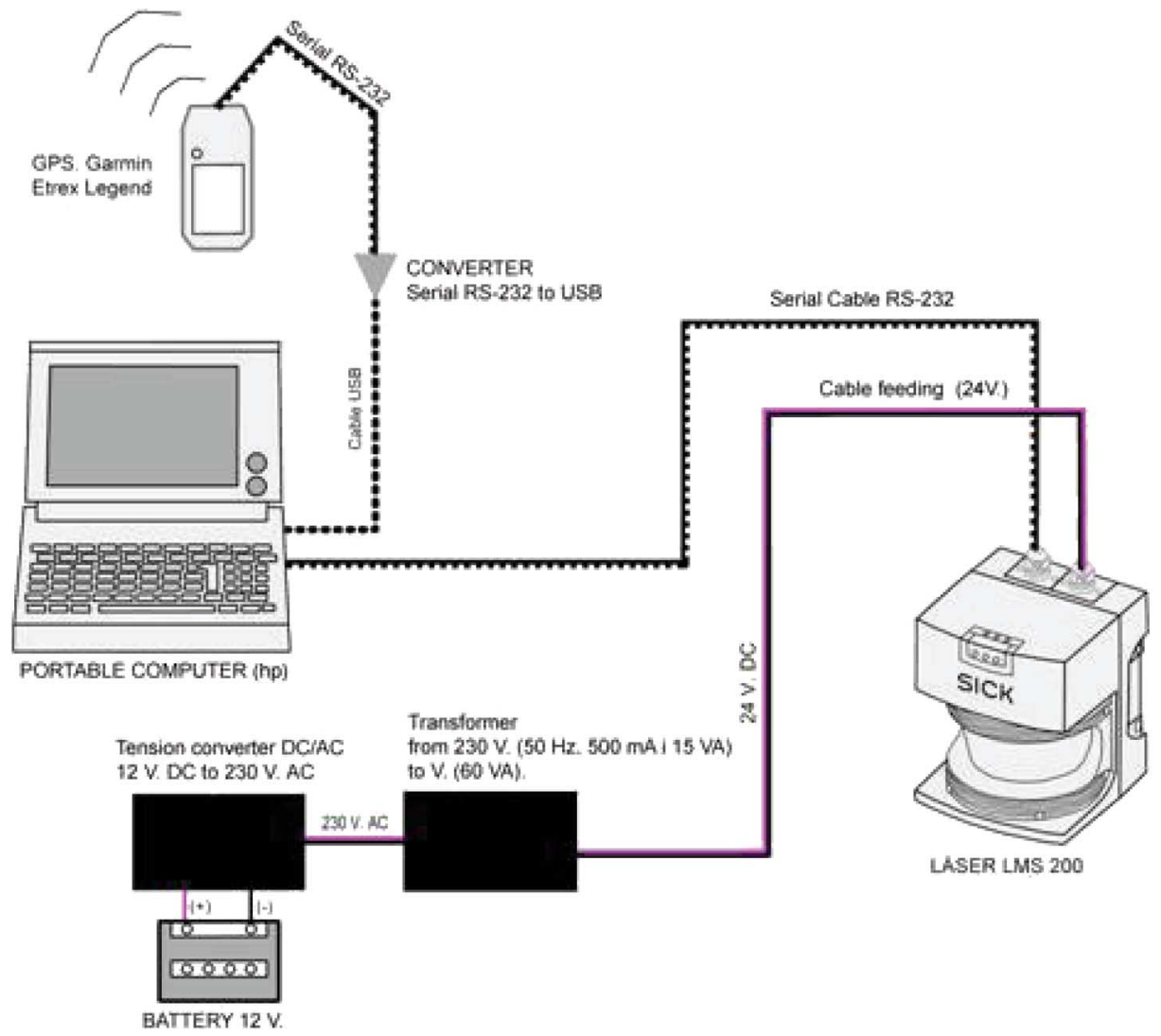

Fig. 3. Electronic equipment of the tree-foliage measurement system.

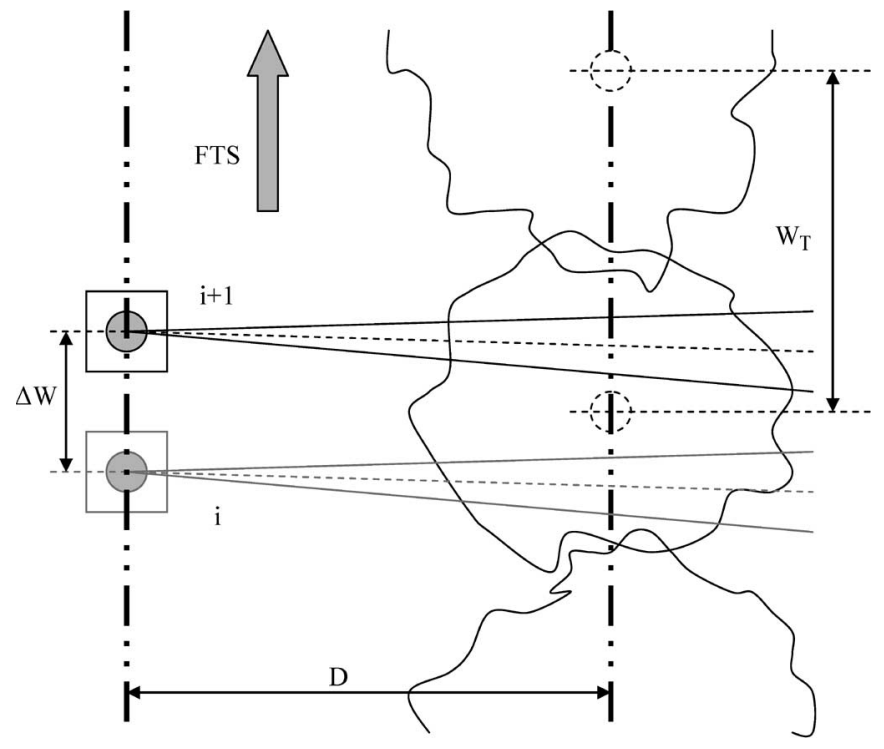

Fig. 4. Top view of the scanning scenario.

$D_{\mathrm{T}} \quad$ distance between two parallel rows of trees;

$H_{\mathrm{S}} \quad$ height of the LIDAR above ground;

$H_{\mathrm{G}} \quad$ height of grass above ground;

$W_{\mathrm{T}} \quad$ distance between two consecutive trees in the tree row line;

$\Delta W$ distance interval between two consecutive vertical scans;

$\Delta t \quad$ time interval between two consecutive vertical scans;

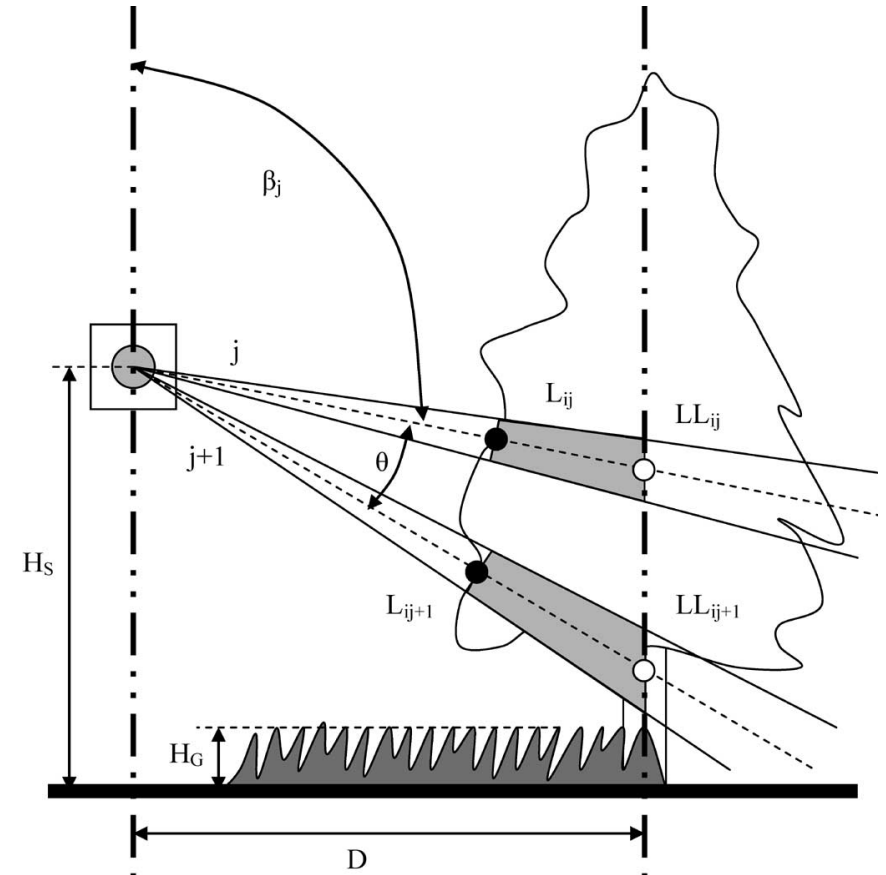

Fig. 5. Front view of the scanning scenario.

$\beta_{\mathrm{j}} \quad$ angle between the vertical direction and laser beam $j\left(\beta_{\mathrm{j}}=j \cdot \theta\right)$;

$L_{\mathrm{ij}} \quad$ distance measured from the LIDAR to the target for slice $i$ and beam $j$;

$L_{\mathrm{ij}}$ distance from the LIDAR to the vertical plane that contains the tree row line. 


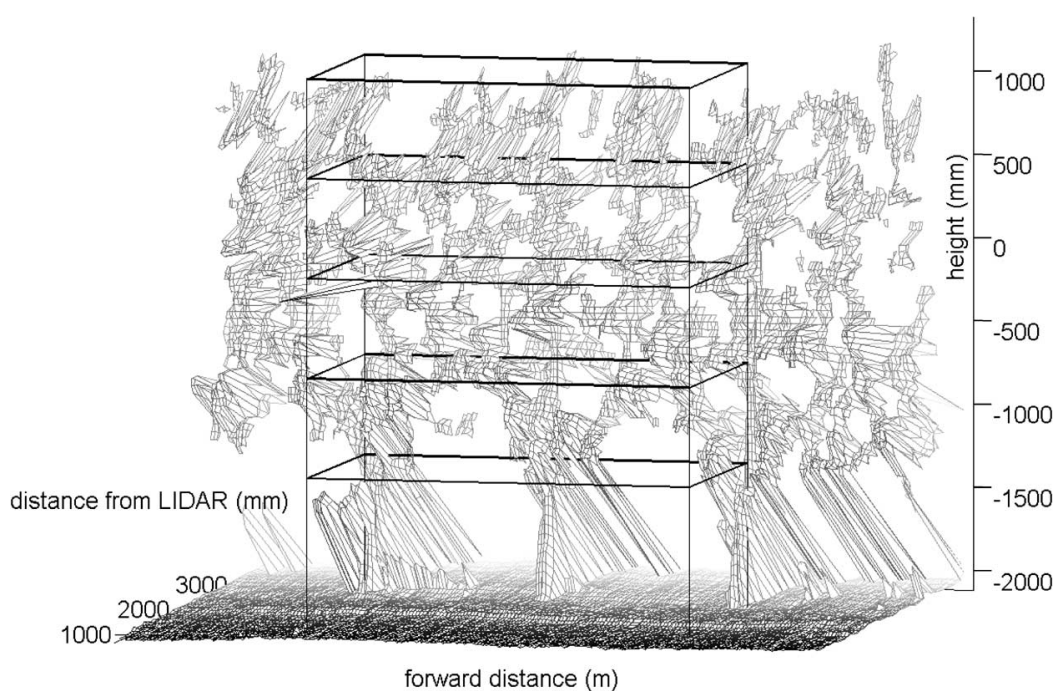

Fig. 6. LIDAR data of a typical pear-tree crop obtained with $H_{\mathrm{S}}=2100 \mathrm{~mm}, D_{\mathrm{i}}=1470 \mathrm{~m}$, FTS $=0.322 \mathrm{~m} / \mathrm{s}$, and $\Delta W=31 \mathrm{~mm}$. The measurement boxes are also plotted for reference.

Only $H_{\mathrm{S}}, \theta$ and the structural parameters of the trees $D_{\mathrm{T}}$, and $W_{\mathrm{T}}$ are known a priori. Fig. 6 shows a mesh representation of the LIDAR data of a pear-tree crop with a representation of the boxes used for calibration.

The final goal is to obtain a relation between the volume and the foliage surface. The volume can be estimated using the data obtained by the LIDAR, but some preprocessing must be applied to the raw data to remove sky points, grass, and other false measurements.

1) Removal of the Sky: The LIDAR was configured with a measurement range of up to $8 \mathrm{~m}$, and sky points and other larger distances are returned as a false measurement of $8 \mathrm{~m}$. Therefore, all these points can be easily removed from the raw data using a radial threshold value of, for example, $7.9 \mathrm{~m}$.

2) Removal of the Grass: The grass and the ground must be removed from the raw data to avoid its inclusion in the computation of the volume. To this end, the height of the grass can be deduced directly because $H_{\mathrm{S}}$ is known, and it is supposed that there is only grass under the LIDAR, for example, the data points corresponding to angles from $170^{\circ}$ to $180^{\circ}$ define a maximum value for $H_{\mathrm{G}}$. This removal is easiest if the polar raw data corresponding to the angles from $90^{\circ}$ to $180^{\circ}$ is converted to rectangular and if a height threshold value is defined. Fig. 7 shows the data identified as a part of the grass or ground corresponding to the raw data presented in Fig. 6.

3) Removal of False Measurements: There are false measurements in the raw data. Most of them are originated by flying objects, small birds, and big insects. The only way to remove some of these false measurements is to analyze some initial slices in order to extract an estimation of the distance where the tree begins; if this distance can be estimated, then all data points located before this distance threshold value must be removed. This procedure is simple and only requires the raw data of one slice to be applied, although removal of false-measurement points before this threshold distance will require the use of 3-D morphological filters applied to several consecutive slices to detect isolated points. Therefore, the simplest procedure was selected in order to enable real-time operation.

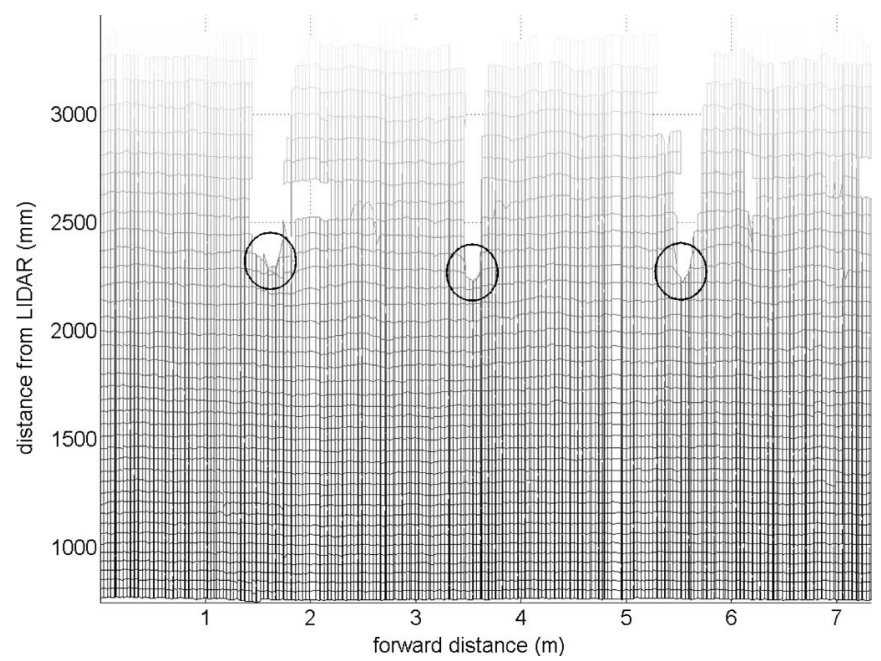

Fig. 7. LIDAR data identified as part of the grass. The position of the trunks is labeled with a circle.

\section{Volume Measurement}

The preprocessed slices can be integrated to obtain the volume $V(k, p)$ of a region of the crop.

$$
V(k, p)=\sum_{i=k}^{p} \sum_{j=0}^{N-1} S_{i j} \cdot \Delta W
$$

where $S_{i j}$ is the surface between the $j$ and $j+1$ laser measurement in the slice $i, k$ and $p$ are the starting and ending travel slices, and $N$ is the number of measurements in one slice. This surface can be computed if the distance to the vertical plane that defines the center of the tree row line $D_{i}$ and the rectangular coordinates $\left[X_{i j}, Y_{i j}\right]$ of each measurement point $L_{i j}$ are available. For example, if $\left(X_{i j} \leq D_{i}\right)$ and $\left(X_{i j+1} \leq D_{i}\right)$

$$
S_{i j}=\frac{\left(2 \cdot D_{i}-\left(X_{i j}+X_{i j+1}\right)\right)}{2} \cdot\left(Y_{i j}-Y_{i j+1}\right) .
$$


A set of different horizontal boxes were defined to have an estimation of the volume and the foliage surface at different heights (Fig. 6). The boxes cover a number of trees because, in a normal grove, the trees are not isolated, and it is not possible to estimate the volume of only one tree. The boxes start exactly in the middle of the tree row line (position of $D_{i}$ ), covering only one side (half) of the trees. Therefore, depending on the relative location of the box and the measurement points $L_{i j}$ and $L_{i j+1}$, a set of special geometric conditions appears in the computation of $S_{i j}$, and the speed of this computation must be carefully improved for real-time operation.

However, the most difficult point is to obtain a good estimation of the distance to the center of the tree for each slice $D_{i}$ because of the uncertainty in the spatial trajectory of the LIDAR. In this particular case, to solve this crucial problem, we propose to detect the position of the trunks in the data discarded as grass or ground (see Fig. 7, circles) and then dynamically estimate $D_{i}$ by linear interpolation between consecutive trunks. This solution is very effective but depends on the height of the grass and is probably also species-dependent.

\section{Foliage-Surface Measurement}

The foliage surface must be measured manually, collecting (removing) the leaves of the trees inside the boxes and then measuring their total one side area using a specialized machine [19]. Obviously, this destructive measurement must be done before a LIDAR scanning and is only required for calibration purposes.

\section{E. Calibration of the Foliage-Surface Estimator}

Finally, Fig. 8 shows the volume and foliage surface obtained in the calibration boxes. The data correspond to five different areas of the same grove, scanned with the LIDAR and then manually defoliated in alternate days. In each area, five boxes have been defined with heights [0,900], [900, 1500], [1500, 2100], [2100, 2700], and [2700,3300] $\mathrm{mm}$ relative to the ground; $1000 \mathrm{~mm}$ large relative to the center-row line of the trees; and different width but, at least, completely covering three consecutive trees.

The coefficient of correlation of the data is 0.81 and 0.86 for a first- and second-order linear fitting, showing that there are structural differences in the trees, depending on their relative location in the grove. Therefore, it is very important that the calibration data (Fig. 8) represent the average relation; the coefficient of correlation for a linear fit is better than 0.98 when using the data set of only one area. In the data shown in Fig. 8, the maximum relative error using a linear estimator is $93 \%$, but the average relative error is below 6\%, which is small enough to allow the use of the proposed measurement system. The maximum relative error mainly originates in the first height level $([0,900] \mathrm{mm})$ because, in this area, the foliage surface is very small, but the volume measured is high (Fig. 8, circle) due to the trunks of the trees.

\section{EXPERIMENTAL VALIDATION}

The experimental evaluation of the proposed measurement system consists of an estimation of the foliage surface of a part

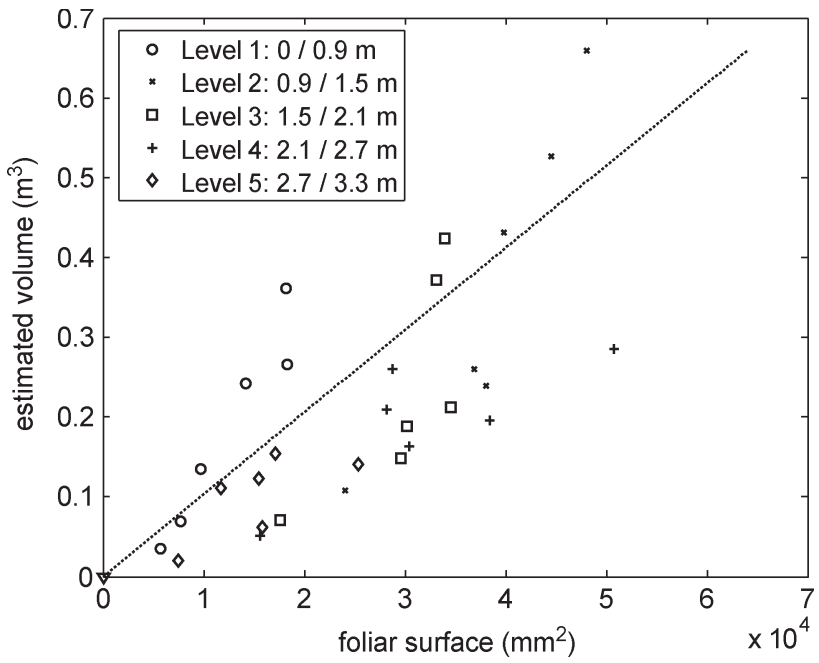

Fig. 8. Relation between volume and foliage surface for pear trees at different heights. The line shows the linear fitting of the data.

TABLE I

RELATIVE ERRor IN THE Estimation OF THE Foliage Surface

\begin{tabular}{|c|c|c|c|c|c|c|}
\hline Height $(\mathrm{m})$ & Case 1 & Case 2 & Case 3 & Case 4 & Case 5 & Average \\
\hline$\left[\begin{array}{lll}2.7 & 3.3\end{array}\right]$ & $-39 \%$ & $-4.3 \%$ & $-32 \%$ & $-32 \%$ & $-14 \%$ & $-26.8 \%$ \\
\hline$\left[\begin{array}{ll}2.1 & 2.7\end{array}\right]$ & $-22 \%$ & $-25 \%$ & $-7.1 \%$ & $-23 \%$ & $-21 \%$ & $-25.7 \%$ \\
\hline$\left[\begin{array}{ll}1.5 & 2.1\end{array}\right]$ & $-5.5 \%$ & $-38 \%$ & $-12 \%$ & $5.8 \%$ & $-4.1 \%$ & $-10.4 \%$ \\
\hline$\left[\begin{array}{ll}0.9 & 1.5\end{array}\right]$ & $6.6 \%$ & $8.9 \%$ & $9.2 \%$ & $0.4 \%$ & $8.2 \%$ & $7.6 \%$ \\
\hline$\left[\begin{array}{ll}0 & 0.9\end{array}\right]$ & $48 \%$ & $72 \%$ & $55 \%$ & $22 \%$ & $93 \%$ & $59.6 \%$ \\
\hline$\left[\begin{array}{ll}0 & 3.3\end{array}\right]$ & $-4.3 \%$ & $-5.4 \%$ & $-2.6 \%$ & $-3.8 \%$ & $1.1 \%$ & $-4.8 \%$ \\
\hline
\end{tabular}

of the grove. To this end, several areas comprising three trees were scanned using the LIDAR attached in a tractor unit. After this scan, the foliage surface of the area was manually measured in order to validate the estimation. At this stage, some important parameters, such as the height of the grass and the distance from the LIDAR to the center of the trees, were controlled and fixed to minimize their influence in the measurements. In this case, the height of the boxes used for validation coincide with the values used for calibration, although in normal operation, these heights will depend on the pesticide machine used (Fig. 1).

The data points of the slices obtained with the LIDAR are integrated to compute a differential volume associated with each slice inside the validation boxes. This volume is then converted in the foliage surface using the linear estimator obtained in the previous section. Fig. 9 shows the foliage surface estimated from the LIDAR data for the five heights selected. The gray level depicts the instantaneous foliage surface estimated for each slice area. Obviously, a typical sprayer or diffuser does not have the horizontal degree of resolution offered by the measurement system. Therefore, a sliding window must be used to estimate the foliage surface covered by the sprayer, although this aspect is not covered in this paper. Finally, Table I shows the relative error obtained in the estimation of the foliage surface of a line of trees, evaluated on different days and in different areas of the same grove.

\section{DISCUSSION}

The proposed measurement system is based on the hypothesis that an entire volume of trees can be interpreted as foliage 


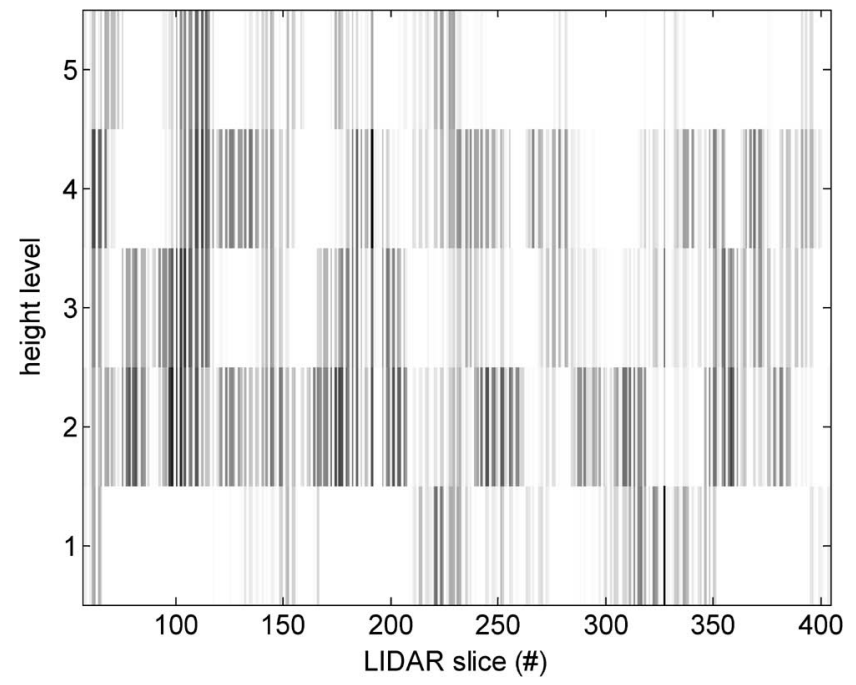

Fig. 9. Estimated absolute foliage surface of a pear-tree crop obtained with $H_{\mathrm{S}}=2100 \mathrm{~mm}, D_{\mathrm{i}}=1470 \mathrm{~m}, \mathrm{FTS}=0.322 \mathrm{~m} / \mathrm{s}$, and $\Delta W=31 \mathrm{~mm}$.

TABLE II

Relative ERRor FOR THE HEIGHT-WeIghted Estimation

\begin{tabular}{|c|c|c|c|c|c|c|}
\hline Height (m) & Case 1 & Case 2 & Case 3 & Case 4 & Case 5 & Average \\
\hline$\left[\begin{array}{lll}2.7 & 3.3\end{array}\right]$ & $-17 \%$ & $30 \%$ & $-7.7 \%$ & $-8.5 \%$ & $16 \%$ & $2.3 \%$ \\
\hline$\left[\begin{array}{lll}2.1 & 2.7\end{array}\right]$ & $9.1 \%$ & $5.0 \%$ & $31 \%$ & $7.9 \%$ & $10 \%$ & $6.8 \%$ \\
\hline$\left[\begin{array}{lll}1.5 & 2.1\end{array}\right]$ & $8.6 \%$ & $-28 \%$ & $0.4 \%$ & $21 \%$ & $10 \%$ & $2.9 \%$ \\
\hline$\left[\begin{array}{lll}0.9 & 1.5\end{array}\right]$ & $-0.8 \%$ & $1.3 \%$ & $1.6 \%$ & $-6.6 \%$ & $0.6 \%$ & $0.1 \%$ \\
\hline$\left[\begin{array}{ll}0 & 0.9\end{array}\right]$ & $-6.6 \%$ & $8.5 \%$ & $-2.5 \%$ & $-22 \%$ & $39 \%$ & $3.2 \%$ \\
\hline$\left[\begin{array}{ll}0 & 3.3\end{array}\right]$ & $1.5 \%$ & $-2.6 \%$ & $7.1 \%$ & $0.7 \%$ & $188 \%$ & $19 \%$ \\
\hline
\end{tabular}

surface. This hypothesis can be accepted for a certain variety of species but not for the one analyzed in this paper because of its long trunk. This effect can be observed in Fig. 8 because the data corresponding to the first height, which is $[0,0.9]$ $\mathrm{m}$, has a slope greater than the other points due to its highvolume and low-foliage surface. This is probably the cause of the poor coefficient of correlation obtained with a linear fitting $R=0.81$. The effect of the trunk is clearly observed in Table I, because the foliage surface is over-estimated at lower heights, whereas it is under-estimated at greater heights.

The best way to avoid the effect of the trunk is to have an initial estimation of the volume of the wood of the trees, for example, with an initial scanning in winter. However, this alternative is currently too complex for a real-time application because, then, all of the slices of the initial scan must be stored and precisely geo-referenced to allow its posterior compensation. Alternatively, we propose the following approach to compensate the trunk volume: Weight the estimation of the foliage surface with the average error values obtained at a different height in Table I. Fig. 10 shows the five weights deduced to compensate the relative error and a second-order fitting to interpolate the correction for other heights. Table II shows the relative error obtained with the height-weighted correction where the average relative error in the estimation of the foliage surface has been reduced for all heights. However, in spite of not having been tested, it is supposed that the proposed improvement is dependent on the species and the height of the tree.

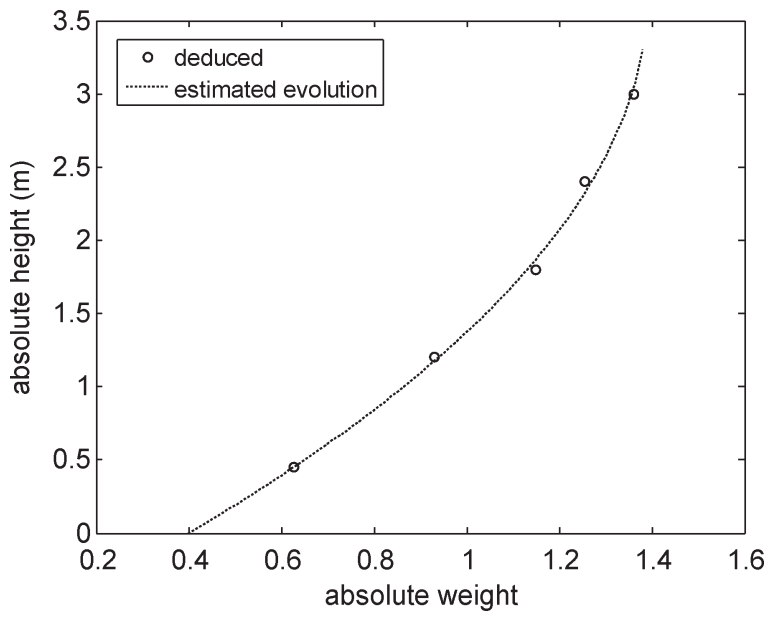

Fig. 10. Values of the weight applied to correct the foliage-surface estimation depending on the height.

Another important aspect is the procedure to estimate the volume from the LIDAR data or, more specifically, $S_{i j}$. In this paper, $S_{i j}$ is computed using the rectangular projection of the data points obtained with the LIDAR, as many other authors do [13], [14]. However, the LIDAR provides a cylindrical projection of the environment, and other methods can be used to compute this intermediate surface.

\section{CONCLUSiON}

In this paper, a measurement system, based on a ground laser scanner, has been proposed and tested for tree-foliage estimation. The foliage surface is estimated through the measurement of the volume that is computed directly from the slices obtained with a LIDAR. The main conclusion is that the relation between the external volume of the tree and its foliage surface can be considered linear with an average relative error of less than $6 \%$ in the estimation for a complete grove, although the trunk originates instantaneous relative errors of up to $93 \%$ in the lower part of the trees. These results can be improved up to $50 \%$ using an additional height-weighted correction.

The simplicity of the proposed method allows length and height-dose control in pesticide and fertilizer machines in realtime and with an acceptable average error in the foliage estimation for a complete grove, which is an improvement over other works, where only the volume or density is estimated.

Nevertheless, several aspects of the measurement method must be studied in depth to improve the accuracy of the estimation, such as the general relation between the foliage volume and surface of a tree, the need for a specific calibration depending on the species measured, the method to compute the volume from the slices, the procedure to estimate and compensate the volume of the trunk, and the procedure to detect the relative position of the tree line.

\section{REFERENCES}

[1] E. C. Hislop, "Can we define and achieve optimal pesticide deposits?" Asp. Appl. Biol., vol. 14, pp. 153-172, 1987, Studies of Pesticide Transfer and Performance. 
[2] I. Hiroaki, N. Toyonori, and T. Eiji, "Measurement of pesticide residues in food based on diffuse reflectance IR spectroscopy," IEEE Trans. Instrum. Meas., vol. 51, no. 5, pp. 886-890, Oct. 2002.

[3] Y.-R. Chen, K. Chao, and M. S. Kim, "Machine vision technology for agricultural applications," Comput. Electron. Agric., vol. 36, no. 2/3, pp. 173-191, 2002.

[4] D. K. Giles, M. J. Delwiche, and R. B. Dodd, "Electronic measurement of tree canopy volume," Trans. ASAE, vol. 31, no. 1, pp. 264-272, 1988.

[5] B. Li, J. D. Whitney, W. M. Miller, and T. A. Wheaton, "Ultrasonic-based canopy volume measurements of citrus trees," presented at the ASAE, St. Joseph, MI, 2001, ASAE Paper 021053.

[6] S. D. Tumbo, M. Salyani, J. D. Whitney, T. A. Wheaton, and W. M. Miller, "Investigation of laser and ultrasonic ranging sensors for measurements of citrus canopy volume," Appl. Eng. Agric., vol. 18, no. 3, pp. 367-372, 2002.

[7] Q. U. Zaman and M. Salyani, "Effects of foliage density and ground speed on ultrasonic measurement of citrus tree volume," Appl. Eng. Agric., vol. 20, no. 2, pp. 173-178, 2004.

[8] A. Wehr and U. Lohr, "Airborne laser scanning-An introduction and overview," ISPRS J. Photogramm. Remote Sens., vol. 54, no. 2/3, pp. 68-82, 1999.

[9] J. Holmgrena and A. Persson, "Identifying species of individual trees using airborne laser scanner," Remote Sens. Environ., vol. 90, no. 4, pp. 415-423, Apr. 2004.

[10] J. C. Ritchie, D. L. Evans, D. Jacobs, J. H. Everitt, and M. A. Weltz, "Measuring canopy structure with an airborne laser altimeter," Trans. $A S A E$, vol. 36, no. 4, pp. 1235-1238, 1993.

[11] M. Nilsson, "Estimation of tree heights and stand volume using an airborne lidar system," Remote Sens. Environ., vol. 56, no. 1, pp. 1-7, Apr. 1996.

[12] P. Walklate, J. Cross, G. Richardson, R. Murray, and D. Baker, "Comparison of different spray volume deposition models using LIDAR measurements of apple orchards," Biosyst. Eng., vol. 82, no. 3, pp. 253-267, Jul. 2002.

[13] J. Wei and M. Salyani, "Development of a laser scanner for measuring tree canopy characteristics: Phase 1. Prototype development," Trans. ASAE, vol. 47, no. 6, pp. 2101-2107, 2004.

[14] J. Wei and M. Salyani, "Development of a laser scanner for measuring tree canopy characteristics: Phase 2. Foliage density measurement," Trans. ASAE, vol. 48, no. 4, pp. 1595-1601, 2005.

[15] R. Sanz, J. Palacin, J. M. Sisó, M. Ribes-Dasi, J. Masip, J. Arnó, J. Llorens, J. M. Vallés, and J. R. Rosell, "Advances in the measurement of structural characteristics of plants with a LIDAR scanner," presented at the Eur. AgEng Conf., Leuven, Belgium, 2004, Paper 277.

[16] LMS 200/211/220/221/291 Laser Measurement Systems: Technical Description, Waldkirch, Germany: Sick AG.

[17] P. Palojarvi, K. Maatta, and J. Kostamovaara, "Pulsed time-of-flight laser radar module with millimeter-level accuracy using full custom receiver and TDC ASICs," IEEE Trans. Instrum. Meas., vol. 51, no. 5, pp. 1102-1108, Oct. 2002.

[18] J. Nissinen and J. Kostamovaara, "Wide dynamic range CMOS receivers for a pulsed time-of-flight laser range finder," in Proc. IEEE Instrum. Meas. Technol. Conf., 2004, vol. 2, pp. 1224-1227.

[19] F. M. Turrell, M. J. Garber, W. W. Jones, W. C. Cooper, and R. H. Young, "Growth equations and curves for citrus trees," Hilgardia, vol. 39, no. 16, pp. 429-445, 1969.

Jordi Palacín (M'95) received the degree in electronic engineering and the $\mathrm{Ph} . \mathrm{D}$. degree from the University of Barcelona, Barcelona, Spain, in 1997 and 2005, respectively.

Since 1998, he has been an Associate Professor and Research Project Leader with the Robotics Group, University of Lleida, Lleida, Spain. His research interests include compact thermal modeling, signal processing, sensors, and robotics.

Tomàs Pallejà received the degree in informatics from the University of Lleida, Lleida, Spain, in 2006, where he is currently working toward the Ph.D. degree with the Department of Informatics and Industrial Engineering.

His main interests are in mobile robotics and human and robotics interaction.
Marcel Tresanchez received the degree in informatics from the University of Lleida, Lleida, Spain, in 2007, where he is currently working toward the Ph.D degree with the Department of Informatics and Industrial Engineering.

His main interests are mobile robotics and wireless networks.

Ricardo Sanz received the degree in agriculture engineering from the Polytechnic University of Barcelona, Barcelona, Spain, in 1992.

Since 1995, he has been an Associate Professor with the University of Lleida, Lleida, Spain. His research interests include characterization of vegetation, laser sensors, and precision agriculture.

Jordi Llorens received the degree in agriculture engineering from the University of Lleida, Lleida, Spain, in 2006, where he is currently working toward the Ph.D. degree with the Department of Agro-Forestry Engineering.

His research interests are in laser sensors and precision agriculture.

Manel Ribes-Dasi received the degree in agriculture engineering and the Ph.D. degree from the University of Lleida, Lleida, Spain, in 1978 and 1996, respectively.

Since 1979, he has been an Associate Professor with the University of Lleida. His research interests include geostatistics and digital-model terrain.

Joan Masip received the degree agricultural engineering from the Polytechnical University of Catalonia, Lleida, Spain, in 1984 and the Ph.D. degree from the Polytechnical University of Valencia, Valencia, Spain, in 1994.

Since 1985, he has been an Associate Professor with the University of Lleida, where he has also been a Researcher with the Precision Agriculture Group since 2001. His research interests include precision agriculture, conveying of agricultural materials, and agricultural mechanization.

Jaume Arnó received the degree in agricultural engineering from the Polytechnical University of Catalonia, Lleida, Spain, in 1990.

Since 1994, he has been an Associate Professor with the University of Lleida, where he has also been a Researcher with the Precision Agriculture Group since 2001. His research interests include precision agriculture, geostatistics, and agricultural mechanization.

Alexandre Escolà received the degree in agricultural engineering from the University of Lleida, Lleida, Spain, in 2001, where he is currently working toward the Ph.D. degree with a thesis on variable-rate technology in sprayapplication techniques.

From 2001 to 2006, he was a Part-time Assistant Professor at the University of Lleida, where he is currently an Associated Professor. His research interest are focused on the application of new technologies in agriculture, such as the sensing of vegetation, variable-rate technologies, automation of processes in agriculture, and precision agriculture in general.

Joan Ramon Rosell received the degree in physics from the University of Barcelona, Barcelona, Spain, in 1986, the Ph.D. degree in physics (nuclear engineering) from the Polytechnical University of Catalonia (UPC), Barcelona, in 1989, and the degree in electrical engineering from the Rovira i Virgili University, Tarragona, Spain, in 1997.

In 1986, he was a University Professor at UPC. Since 1992, he has been a University Professor at the University of Lleida, Lleida, Spain. His research interests include precision agriculture, sensors, automation, and energy. 